\title{
Comparison of high-resolution magnetic resonance imaging and micro-computed tomography arthrography for in-vivo assessment of cartilage in non-human primate models
}

\author{
Kevin Tse Ve Koon ${ }^{1}$, Denis Grenier ${ }^{1}$, Fabrice Taborik ${ }^{2}$, Anne-Laure Perrier $^{3} \wedge$, Laurent Mahieu-Williame ${ }^{1 \wedge}$, \\ Luc Magnier ${ }^{4}$, Thomas Chuzel ${ }^{4}$, Hugues Contamin $^{2}$, Emmanuel Chereul $^{4} \wedge$, Olivier Beuf ${ }^{1} \wedge$ \\ ${ }^{1}$ Univ. Lyon, INSA-Lyon, Université Claude Bernard Lyon 1, UJM-Saint Etienne, CNRS, Inserm, CREATIS UMR 5220, U1294, Lyon, France; \\ ${ }^{2}$ CYNBIOSE, Marcy-l'Étoile, France; ${ }^{3}$ Univ. Grenoble Alpes, Univ. Savoie Mont Blanc, CNRS, LOCIE, Chambéry, France; ${ }^{4}$ VOXCAN, Marcy- \\ l'Étoile, France
}

Correspondence to: Kevin Tse Ve Koon. Univ. Lyon, INSA-Lyon, Université Claude Bernard Lyon 1, UJM-Saint Etienne, CNRS, Inserm, CREATIS UMR 5220, U1294, CREATIS Bâtiment Léonard de Vinci, 21 Avenue Jean Capelle Ouest, 69616 Villeurbanne Cedex, F69621 Lyon, France. Email: kevin.tsevekoon@creatis.univ-lyon1.fr.

\begin{abstract}
Background: Non-human primate (NHP) could be an interesting model for osteoarthritis (OA) longitudinal studies but standard medical imaging protocols are not able to acquire sufficiently highresolution images to depict the thinner cartilage (compared to human) in an in vivo context. The aim of this study was thus to develop and validate the acquisition protocols for knee joint examination of NHP using magnetic resonance imaging (MRI) at $1.5 \mathrm{~T}$ and X-ray micro-computed tomography arthrography $(\mu \mathrm{CTA})$.

Methods: The first phase of the study focused on developing dedicated in vivo HR-MRI and $\mu$ CTA protocols for simultaneous acquisitions of both knee joints on NHP. For MR, a dedicated two-channel receiver array coil and acquisition sequence were developed on a $1.5 \mathrm{~T}$ Siemens Sonata system and tuned to respect safety issues and reasonable examination time. For $\mu \mathrm{CTA}$, an experimental setup was devised so as to fulfill similar requirements. The two imaging protocols were used during a longitudinal study so as to confirm that repeated injections of loxaglic acid (contrast agent used for $\mu$ CTA) didn't induce any bias in cartilage assessment and to compare segmentation results from the two modalities. Lateral and medial cartilage tibial plateaus were assessed using a common image processing protocol leading to a $3 \mathrm{D}$ estimation of the cartilage thickness.

Results: From HR-MRI and $\mu \mathrm{CTA}$ images, thickness distributions were extracted allowing for proper evaluation of knee cartilage thickness of the primates. Results obtained in vivo indicated that the $\mu \mathrm{CTA}$ protocol did not induce any bias in the measured cartilage parameters and moreover, segmentation results obtained from the two imaging modalities were consistent.

Conclusions: MR and $\mu$ CTA are valuable imaging tools for the morphological evaluation of cartilage in NHP models which in turn can be used for OA studies.
\end{abstract}

Keywords: Cartilage; non-humane primate model; magnetic resonance imaging (MRI); X-ray micro-computed tomography; arthrography

Submitted Jan 14, 2020. Accepted for publication Oct 23, 2020.

doi: 10.21037/qims-20-116

View this article at: http://dx.doi.org/10.21037/qims-20-116

\footnotetext{
^ ORCID: Kevin Tse Ve Koon, 0000-0001-9780-8337; Anne-Laure Perrier, 0000-0002-4698-2375; Laurent Mahieu-Williame, ORCID: 0000-0003-3990-8131; Emmanuel Chereul, 0000-0002-9309-9665; Olivier Beuf, 0000-0002-4516-4565.
} 


\section{Introduction}

Osteoarthritis (OA) is a disease with increasing prevalence with ageing populations in western countries (1). Destruction and loss of articular cartilage are thought to be major elements involved in the pathophysiology of OA (2), however, it is still not clear whether this precedes, accompanies or is a consequence of changes in other tissues such as subchondral bone. One difficulty in detecting these changes in articular cartilage is the lack of nerves within and therefore damages can be painless (3) rendering early detection and treatments even more difficult. Actually, no therapeutic options are available apart from symptomatic relief through either exercise prescriptions (4) or drugs with questionable long-term effects (5).

Symptomatic knee OA in particular affects about $6 \%$ of adults above 30 years old (2) and more than $13 \%$ of persons under 60 years (6), with an estimated lifetime risk of developing it of nearly 50\% (7) and rising with higher BMI and age. Detection is mainly carried out through imaging the knee joint (8-10) with recognized radiographic markers being joint space narrowing, presence of osteophytes, subchondral cysts and sclerosis. Among the different imaging modalities, conventional radiography is the most widespread but is limited mainly due to positioning issues, lack of direct visualization of articular cartilage (forbidding access to the physiopathology of OA), 2D images display multiple overlapping structures and last but not least the lack of sensitivity of the technique which hinders early detection. NMR techniques are major alternatives to conventional radiography (11-16) and magnetic resonance imaging (MRI) in particular due to its exquisite contrast, spatial resolution, 3D anatomical images and morphometric assessments. Moreover, multiple MRI-based techniques (T1-rho, T2 mapping) give access to different information on structure and content of cartilage related to the progress of OA (16). Any potential medical treatment is bound to be more successful if taken during the earlier stages. However, this is hard to be applied to humans since early detection of OA is a major issue. Subsequently, works have focused on animal models such as mice (17), rats (18-20), guinea pigs $(21)$, rabbits $(22,23)$ and non-human primates (NHPs) (24-29) whereby OA could be induced.

Small animal models of OA do not mimic perfectly the complex conditions occurring in human $\mathrm{OA}$ which is the case for OA in NHP thus representing a useful model. However thinner cartilage in NHP requires higher image resolution for detection of radiographic OA, an essential step before considering more advanced imaging techniques. In the case of MRI, this can be done at the cost of increased scan times leading to a trade-off between scan times and signal to noise ratio (SNR). Use of dedicated coils for knee imaging of NHP can greatly contribute to this end but no such commercial coil suited for NHP is available which has possibly hindered further studies on this model. Another possible imaging modality is $\mathrm{X}$-ray micro-computed tomography which can provide even higher spatial resolution and enables both cartilage and bone assessment. However, knee cartilage imaging requires injection of a contrast agent before application and this injection procedure may generate either a damage to the cartilage and/or a bias in the cartilage thickness determination in the acquired images which therefore needs to be looked into specially in an in vivo context.

A recent study (30) has tackled multimodal imaging (MRI and high-resolution peripheral quantitative computed tomography) of cadaveric knees but to the best of our knowledge none has tackled knee or cartilage characterization through in vivo imaging of a NHP model although this model is a very interesting one with respect to OA. The present study was geared towards:

* Developing a protocol (setup and sequence) that will yield reproducible high-resolution MR images of knee joints in NHP models and thus help in the development of more advanced MRI studies.

* Developing a protocol (setup and contrast agent protocol) to acquire micro-computed tomography arthrography ( $\mu \mathrm{CTA}$ ) images of the knee joints in the same NHP models and cross-checking the absence of impact of the procedure on cartilage characterization.

* Evaluating the potentialities of $\mu$ CTA by comparing cartilage layer thicknesses quantified through both imaging modalities.

\section{Methods}

The first phase of this study focused on the development of a HR-MRI protocol for knee joint examination of NHP on a $1.5 \mathrm{~T}$ Siemens-Sonata system. It enabled simultaneous acquisition of high-resolution images of both knee joints within a time frame respectful of animal ethics. Upon validation, six primates were enrolled in this preliminary longitudinal study where HR-MRI and X-ray $\mu \mathrm{CTA}$ were performed to assess the eventual impact of the $\mu$ CTA protocol (repeated injection of loxaglic acid) on 
A

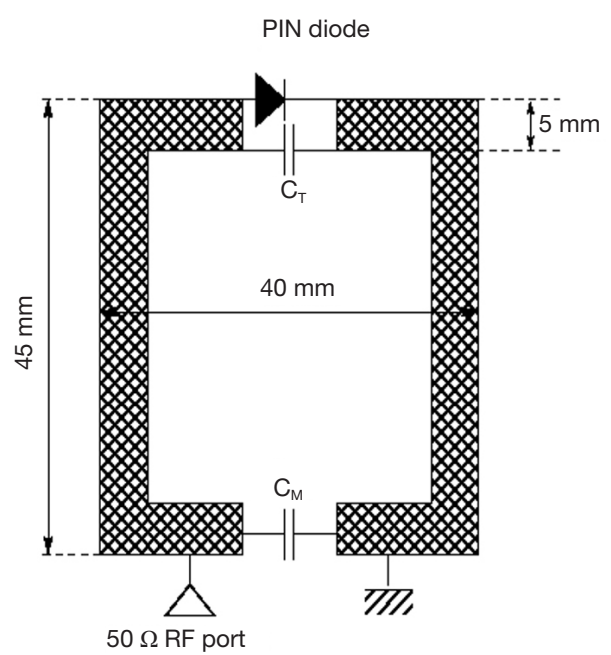

B

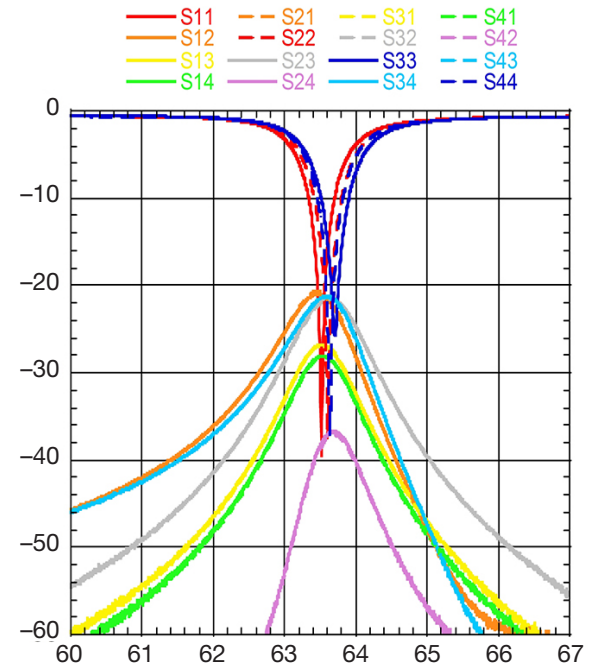

Figure 1 (A) Coil geometry and (B) reflection and transmission (S) parameters of the two channel array coils in experimental configuration.

the morphologic evaluation of cartilage parameters of the medial and lateral tibial plateaus. This evaluation was carried out through a double segmentation (manual followed by an automatic regional) procedure of the acquired images, yielding segmented medial and lateral tibial cartilage regions of interests (ROIs). Inside these ROIs, the quantification of cartilage thickness was performed using the method developed by Hildebrand et al. (31) giving 3D thickness parameter distributions characterizing each ROI. These distributions were then statistically tested (one-way ANOVA run on XLSTAT) for differences of their means. Obtained mean thicknesses of the lateral and medial tibial cartilages have also been compared using Bland-Altman analyses.

\section{MRI acquisition protocol}

To obtain suitable MR images, a homemade receiver coil was developed targeting maximum SNR with the best signal uniformity. In its final version (32) the coil was made up of two channels each consisting of a rectangular copper track coil having internal dimensions $30 \mathrm{~mm} \times 35 \mathrm{~mm}$ and etched on a flexible $508 \mu \mathrm{m}$ thick substrate (RogersTM RT/Duroid 5880) which was in turn etched on a rigid cylindrical tube fitting the NHP knee as shown on Figure $1 A . \mathrm{C}_{\mathrm{T}}$ and $\mathrm{C}_{\mathrm{M}}$ are two CMS capacitors with respective values of $620 \pm 20 \mathrm{pF}$ and $100 \pm 2 \mathrm{pF}$ while the PIN diode was a DH80055 one. Optimal coil overlapping was sought so as to minimize coupling between the two elements as shown in Figure $1 B$
$\left(\left|\mathrm{S}_{\mathrm{nm}}\right|\right.$ parameter $\left.<-20 \mathrm{~dB}\right)$. A coil configuration file was created to drive the MR system interface in array mode. Experimental characterization (signal uniformity and SNR) was performed on cylindrical phantoms filled with $1.25 \mathrm{~g} / \mathrm{L}$ of $\mathrm{NiSO}_{4}$ and $5 \mathrm{~g} / \mathrm{L}$ of $\mathrm{NaCl}$ solution. SNR was calculated by taking the ratio between mean signal intensity inside the coil volume in the ROI corresponding to cartilage region and standard deviation (SD) outside. Signal uniformity is taken to be the SD of the signal intensity inside the coil in the ROI as shown in Figure 2. This homemade coil was compared to the Siemens small loop coil (FLS) which was the only available commercial solution for our purpose at our facility.

During acquisitions on primates, one dual-channels array coils was used per knee, using two flex interfaces from Siemens. The primates were in supine position with both legs positioned inside a foam having a susceptibility similar to tissue values (33). Unless this foam was used, the shimming process did not yield proper results (34). Both dual array coils were placed on top of patella to encompass the whole knee joint (Figure 3). A minimum distance of $100 \mathrm{~mm}$ between both knees was kept to ensure at least $20 \mathrm{~dB}$ decoupling between the two elements located at medial sides of each coil. HR-MRI was performed in the sagittal plane using a $3 \mathrm{D}$ water excitation FLASH sequence: $25^{\circ}$ flip angle, TR/TE $=27 / 11.7 \mathrm{~ms}, 70 \mathrm{~Hz} /$ Pixel receiver bandwidth. A total of 120 partitions $(220 \mu \mathrm{m}$ thick) were acquired with a FOV of $50 \times 50 \mathrm{~mm}$ and an in-plane pixel of $112 \times 131 \mu^{2}$. Scan time was $20 \mathrm{~min}$ for sequential 


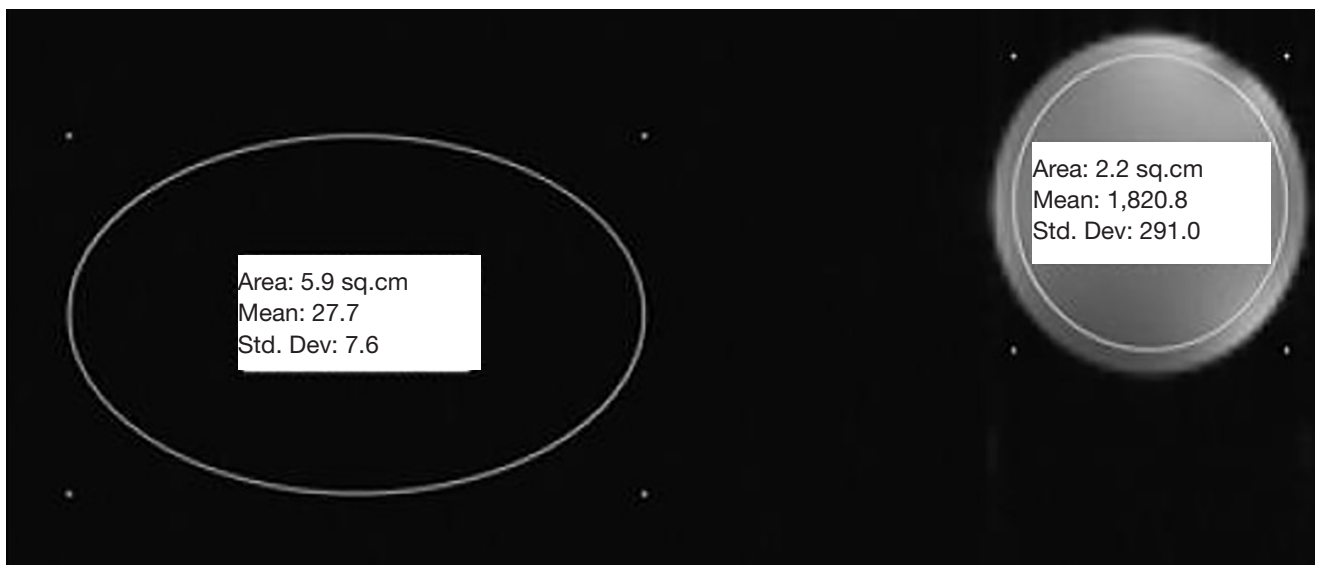

Figure 2 MRI images of a water phantom used for SNR characterization of the developed receiver coils. MRI, magnetic resonance imaging; SNR, signal to noise ratio.

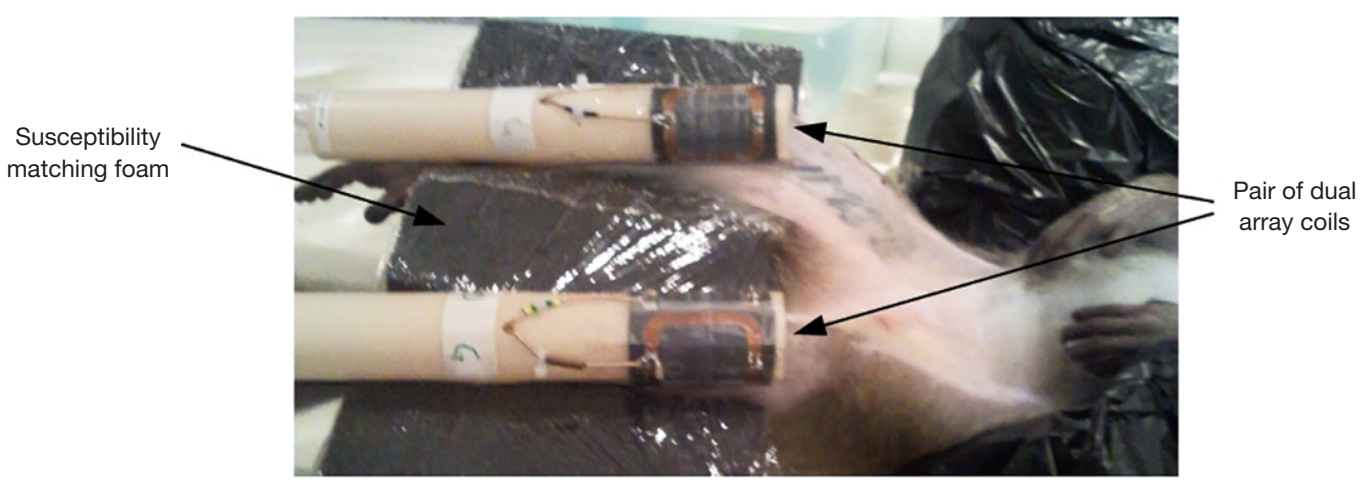

Figure 3 Experimental setup for MRI acquisitions. MRI, magnetic resonance imaging.

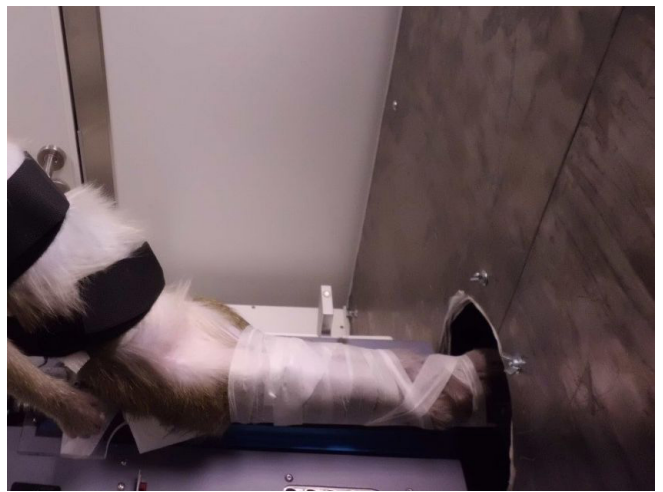

Figure 4 Experimental setup for $\mu$ CTA acquisitions. $\mu$ CTA, microcomputed tomography arthrography. acquisitions of both knees.

\section{$\mu C T A$ acquisition protocol}

To clearly distinguish the cartilage, injection of a contrast agent was mandatory; $2 \mathrm{~mL}$ loxaglic acid $(320 \mathrm{mgI} / \mathrm{mL})$ with 40/60 dilution in PBS was injected in the synovial capsule using a $23 \mathrm{G}$ needle. $\mu \mathrm{CTAs}$ were performed on a GE Locus $\mu$-CT at standard voltage $(80 \mathrm{kV})$ and amperage $(450 \mu \mathrm{A})$ parameters with an isotropic resolution of $90 \mu \mathrm{m}$. Simultaneous acquisition of both knees was done in 15 minutes. Figure 4 shows the positioning of the knees inside the $\mu \mathrm{CT}$ scanner requiring a specific seating setup. 


\section{Study protocol}

All animal studies and experiments were performed under project license $\mathrm{N}^{\circ} 1077$ granted by the Institutional Animal Care and Use Committee (IACUC) of VetAgroSup in compliance with the European regulation on animal experimentation (Directive 2010/63/EU) and its national transposition. Every effort was done to minimize animal suffering and reduce the number of animals used in the experiments.

A total of six young (average age of 4 years at the beginning of the study, weights ranging between 3.2 and $4.3 \mathrm{~kg}$ at the end of the study) female cynomolgus monkeys (Macaca fascicularis) were examined during this phase of the study. Animals were acclimated for at least 10 days prior to the first day of study, and were housed collectively on a natural bedding with the following ambient parameters: ventilation with $>10$ air changes/hour, and no air recirculation, 12-hour light/12-hour dark photoperiod, room temperature of $22 \pm 4{ }^{\circ} \mathrm{C}$, and a humidity of $55 \% \pm 20 \%$. Animal room and enclosure cleaning was performed on a daily basis. They were fed with standard diet (Ssniff Pri V3944-000); food ration was adapted for each individual according to its body weight (100 g per animal per day under $5 \mathrm{~kg} ; 200 \mathrm{~g}$ over $5 \mathrm{~kg}$ ). One fruit was provided daily to each animal. Delicacies were also given in the bedding at the end of the day to allow foraging, as part of the testing facility environmental enrichment program.

The primates were assessed using both HR-MRI and $\mu \mathrm{CTA}$ imaging of the knee joints (lateral and medial tibial plateaus). The latter modality requires injection of a contrast agent in the synovial capsule (10 knee flexionsextensions were performed post-injection). One of the goals of the study was thus to assess the eventual impact of repeated injection of loxaglic acid. Only the right knee underwent $\mu$ CTA imaging while HR-MRI (MR acquisitions of the right knee were carried out after $\mu \mathrm{CTA}$ acquisitions) was applied to both knee joints and several examinations were scheduled at baseline, 30, 60, 90 and 180 days. The primates were fasted at least 12 hours for food and 2 hours for water before starting the anaesthetic procedure. For HR-MRI exam, induction of anaesthesia was performed by intramuscular (IM) injection of ketamine $(15 \mathrm{mg} / \mathrm{kg})$ and midazolam $(1.25 \mathrm{mg} / \mathrm{kg})$. Around 1 hour after the first injection, maintenance of anaesthesia was performed by IM injection of ketamine $(7.5 \mathrm{mg} / \mathrm{kg})$ and midazolam $(0.63 \mathrm{mg} / \mathrm{kg})$. If necessary, a third injection (same agents and same doses as previously) could be used to increase acquisition time. For $\mu \mathrm{CTA}$ exam, induction of anaesthesia was performed by IM injection of ketamine $(10 \mathrm{mg} / \mathrm{kg})$ and midazolam $(1 \mathrm{mg} / \mathrm{kg})$. An endotracheal tube was inserted into the trachea and anaesthesia was maintained with isoflurane $(0.5 \%)$ in oxygen $(100 \%$, $1 \mathrm{~L} / \mathrm{min})$. For both protocols, physiological vitals of the animals were monitored (temperature, breathing rate, blood pressure and heart rate).

Two of the primates presented missing data since HRMR images presented severe artifacts due to unknown reasons. This rendered segmentation impossible (both for left knee at D180). Otherwise, for all other primates the image processing procedure could be applied at all time steps.

\section{D thickness parameter extraction procedure}

So as to suppress an inter-observer variability, images from both modalities were processed by the same operator using the GE Locus $\mu$-CT Microview software version ABA2.2:

* Image pre-processing step: image smoothing using an anisotropic filtering (adaptive threshold for each modality but identical for all monkeys within each modality) enabled noise attenuation in the images and better cartilage frontier visualization.

* Segmentation step: acquired stacks of slices were sampled; about 1 out of every $4-5$ slices. These were processed to obtain 3D thicknesses of the lateral and medial cartilage tibial plateaus. A manual contour segmentation was performed to roughly isolate the cartilage in the ROIs, followed by a regional automatic global segmentation extracting accurately the morphology of both medial and lateral cartilage ROIs. The thresholds used in this automatic procedure were modality dependent but the same for all animals. A manual correction was sometimes applied if the automatic segmentation was not appropriate. When this was not possible for a given slice, an extrapolation was carried out using the two adjacent slices.

* Quantification step: inside the segmented cartilage ROIs, the quantification of cartilage thicknesses was performed following the method described by Hildebrand et al. (31). For each pixel p found inside the $3 \mathrm{D}$ segmented region $\Omega$, the local thickness $\tau(\mathrm{p})$ is calculated as the diameter of the largest sphere inscribed inside $\Omega$ and containing p: 


$$
\tau(p)=2 \cdot \max (\{r \mid p \in \operatorname{sph}(x, r) \subseteq \Omega, x \subseteq \Omega\})
$$

$x$ and $r$ are respectively the center and radius of the spheres which had sizes quantized by steps of $90 \mu \mathrm{m}$ (the voxel size in $\mu-C T)$.

Morphological results were compared in terms of distribution of the $3 \mathrm{D}$ thickness parameters obtained for each cartilage compartment. The spread of the thickness distribution as well as mean and maximum-values characterize the cartilage compartment. The mean thickness of the structure is defined as:

$$
\bar{\tau}=\frac{1}{\operatorname{Vol}(\Omega)} \iiint \tau(p) d^{3} p ; \operatorname{Vol}(\Omega)=\iiint d^{3} p
$$

where the triple integral is taken over the $3 \mathrm{D}$ segmented region $\Omega$.

The term mean thickness will be used when describing the term defined in Eq. [2] while the term average will be used when considering an average over multiple measurements (at different time points or different primates). The maximum thickness of a cartilage compartment is defined as the diameter of the biggest sphere obtained through the described procedure.

Mean thicknesses of the lateral and medial tibial cartilage have also been compared using Bland-Altman analyses:

(I) Comparisons on the left knee at different time points will give us an insight on the reproducibility of the MRI acquisition protocol and image processing;

(II) Data on the right knee obtained through MRI will enable evaluation of the eventual impact of the injections of the $\mu \mathrm{CTA}$ contrast agent on the cartilage thickness;

(III) Comparisons of $\mu \mathrm{CTA}$ data at different time points enable evaluation of the reproducibility of the $\mu \mathrm{CTA}$ acquisition protocol and image processing;

(IV) And finally mean thicknesses obtained through MRI and $\mu$ CTA will enable comparison of the two modalities.

The results of the Bland-Altman comparisons are summarized in terms of confidence intervals (CIs) of the biases and CIs of the differences between paired values. A $\mathrm{CI}$ of the bias including zero and in the same manner, a CI for the differences under $90 \mu \mathrm{m}$ are signs of concordance between measurements.

It is also interesting to compare the different distributions obtained in a similar manner to what has been done with mean thicknesses. To do so, one-way
ANOVAs with a significance level set to 0.05 where the null hypothesis is that the distributions have equal means (hereby mean refers to the mean of the distribution which is different to the mean thickness) and Tukey's post-hoc analysis were performed. It should be noted that here the mean of the distribution is related to but not equal to the mean thickness defined by Eq. [2]. Three different cases will be distinguished: (I) a $\mathrm{P}$ value above the significance level which points to an absence of difference between the means of the distributions, (II) a $\mathrm{P}$ value under the significance level but with a CI for the difference of the means under $90 \mu \mathrm{m}$ which will be considered as an absence of difference since in the quantification procedure, the bin size is equal to $90 \mu \mathrm{m}$. (III) a P value under the significance level but with a CI above or equal to $90 \mu \mathrm{m}$.

\section{Results}

\section{MR images}

Comparative SNR tests were carried out on the $\mathrm{NaCl}$ phantom between the Siemens small loop coil (FLS) and our homemade double channel coil (DC). The FLASH sequence used during the whole protocol displayed an SNR of DC 4.7 times higher than that of the FLS for a decrease in signal uniformity (SD inside the ROI) of about 1.4. In vivo acquisitions on knee joints of primates using the HRMRI protocol yielded high spatial resolution images of the cartilage (Figure $5 A, B)$.

\section{$\mu C T A$ images}

An example of $\mu$ CTA images obtained is shown in Figure 5C, where the cartilage appears as a hyposignal region surrounded by the subchondral bone and the inside of the synovial capsule in hypersignal intensity. The slicesampling previously mentioned enabled the operator to avoid the rare slices where the contrast agent had not properly diffused inside the synovial capsule.

\section{Segmentation results and reproducibility of MRI protocol}

Figure 6 shows an example of manual and automatic segmentations that have been carried out on the MR images (top row) and $\mu$ CTA images (bottom row). From the segmented images, quantifications are carried out yielding 3D thickness parameter distribution from MR images of both left tibial cartilage compartments and specifically, 

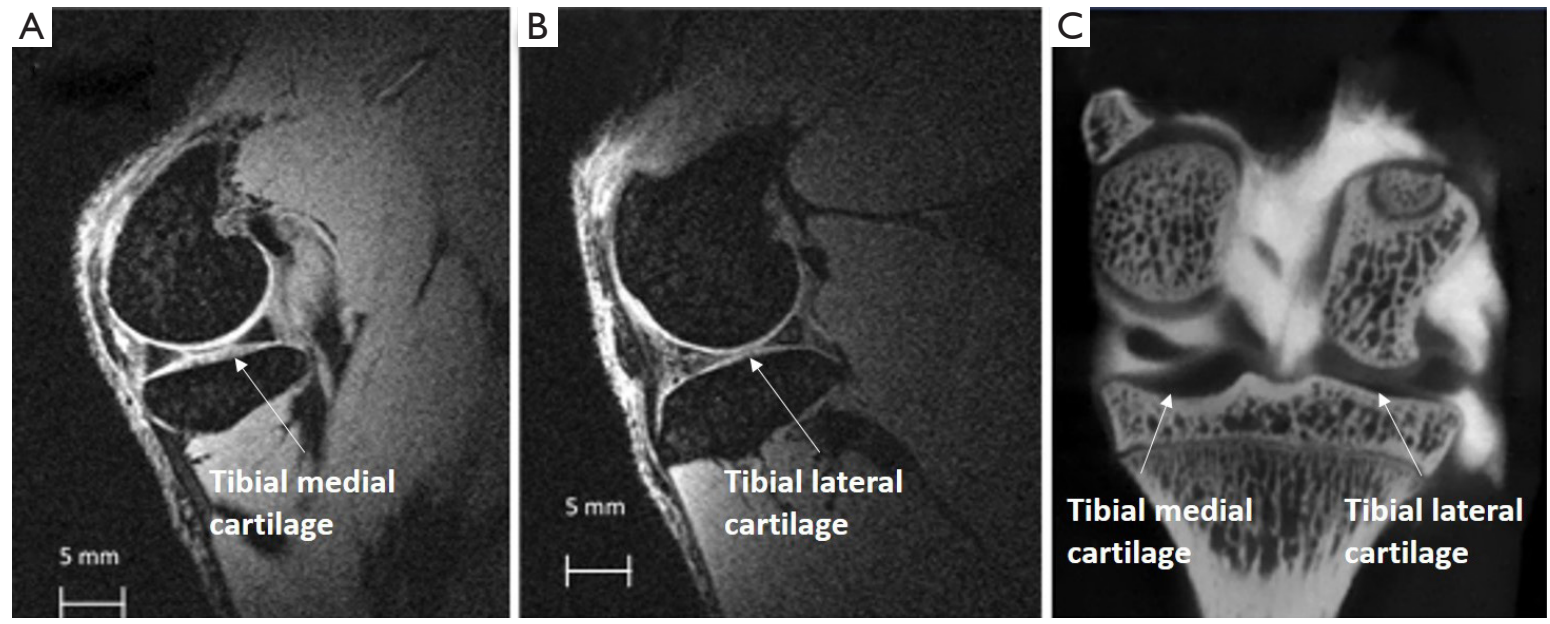

Figure 5 Acquired images: Sagittal HR-MR images acquired on the right knee of a primate (A) medial and (B) lateral side and (C) coronal image or the right knee obtained from $\mu \mathrm{CTA}$ acquisitions. $\mu \mathrm{CTA}$, micro-computed tomography arthrography.
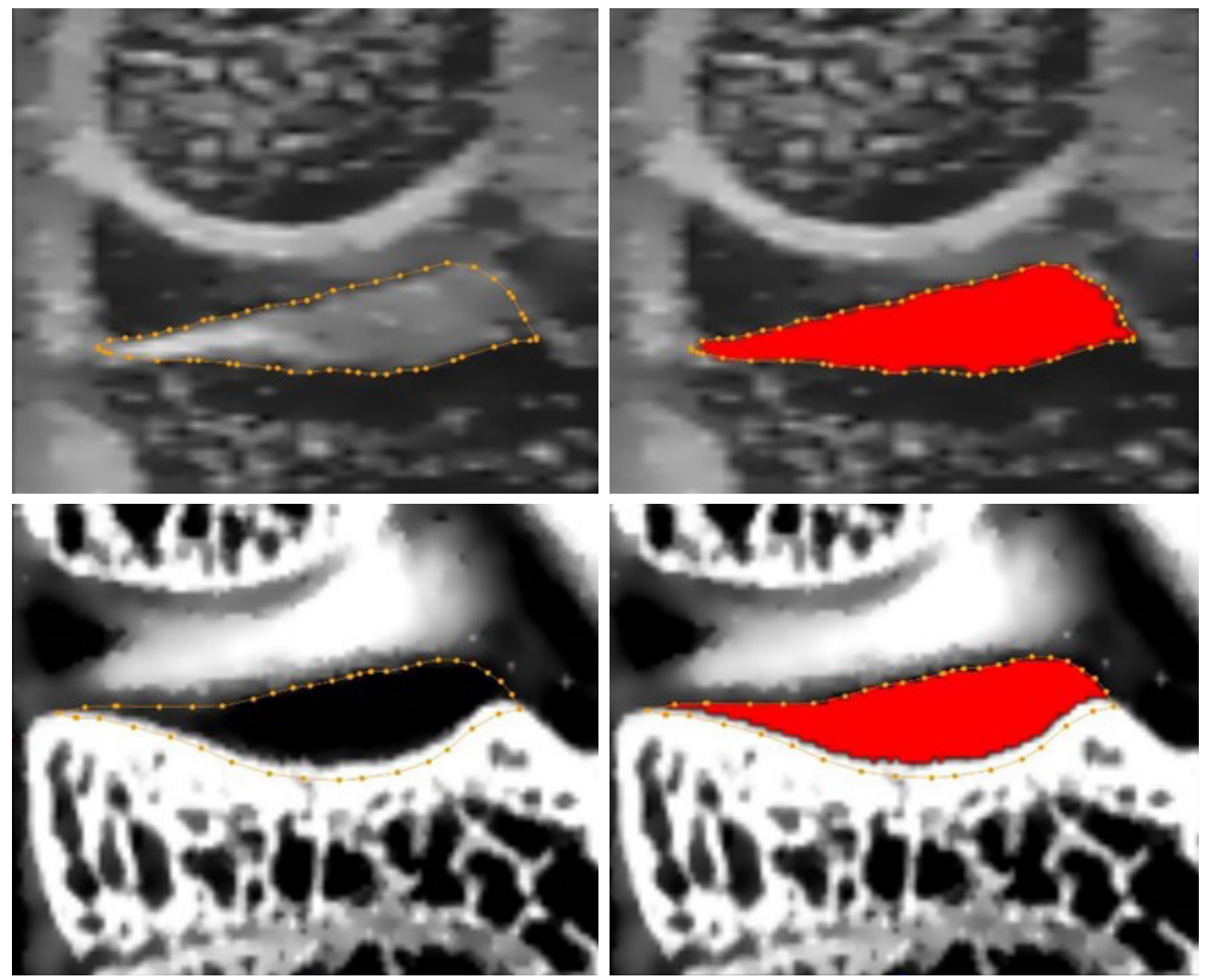

Figure 6 Results of manual (left column) and automatic (right column) segmentations of the tibial cartilage from MRI (top row) and $\mu$ CTA (bottom row) images of the knee. MRI, magnetic resonance imaging; $\mu \mathrm{CTA}$, micro-computed tomography arthrography. 

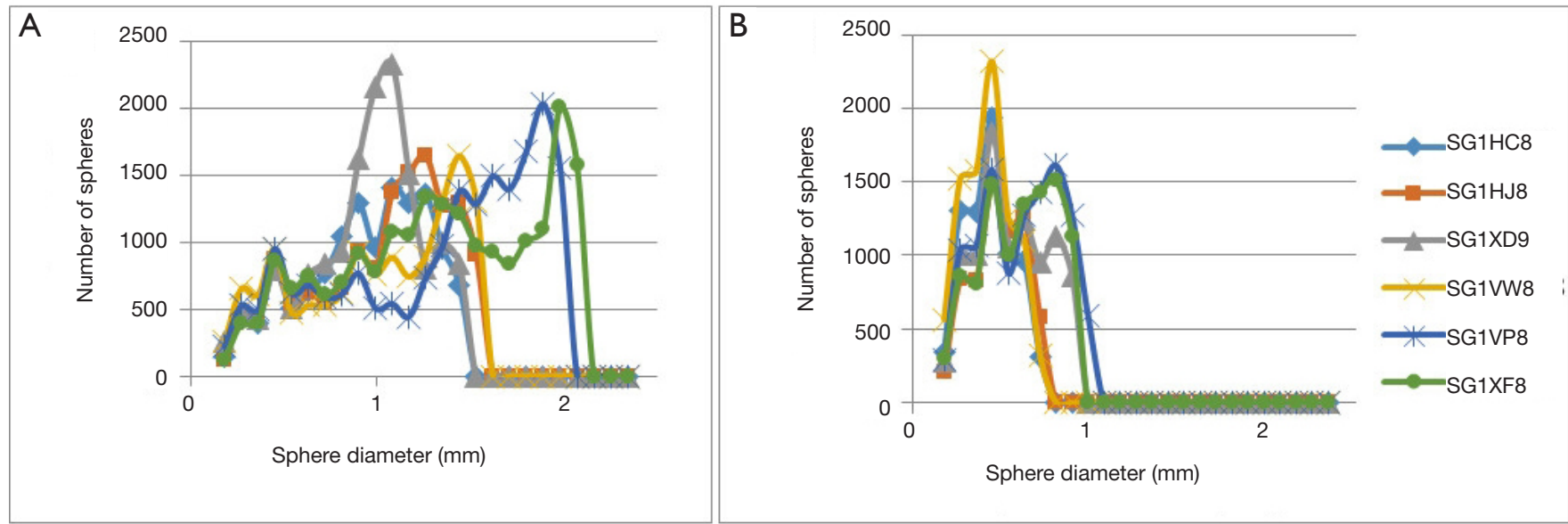

Figure 7 3D thickness parameter distributions obtained through the quantification procedure: (A) left medial and (B) left lateral cartilage compartment, using segmented HR-MR images obtained on all primates at D0.

Figure $7 A, B$ are those obtained on all six primates at D0. Only the tibial plateau was studied because in spite of the dedicated setup, the femoral plateau was too thin with respect to our image resolution, to be considered for image processing. It can be noticed that two of the primates (SG1VP8 and SG1XF8) display higher maximum thicknesses in both compartments.

From the obtained thickness distributions, standard measures such as mean $\{\mathrm{Eq}$. [2] $\}$ and maximum thicknesses of each tibial compartment were extracted. In Figure 8, the time-based average of the mean thickness of left and right, medial and lateral tibial cartilage of all primates are reported for both imaging modalities. The displayed error bars correspond to the calculated SDs obtained from data over the five-time steps. Maximum relative SD for the left compartment was of $10 \%$.

To assess the reproducibility of the determined mean thicknesses at different time points on each primate, BlandAltman analyses were carried out by comparing the obtained values for the left knee at different time points through MRI. An example obtained at D0 and D90 is shown in Figure 9 whereby the full blue line represents the estimated mean bias, the dashed blue line the $95 \%$ CI for the bias and the dashed red line the $95 \%$ CI for the differences between paired values. To summarize the ten Bland-Altman plots (five time points were acquired), in Table 1 is given the $95 \%$ CIs for the bias $\left(^{*}\right)$ and the differences between paired values $\left({ }^{*}\right)$. As can be seen nine out of the ten CI for the bias include zero and only the comparison between D60 and D90 yields a CI excluding zero. As for the CI for the difference three of them display CI above $0.09 \mathrm{~mm}$ which is the bin size of the quantification procedure.

It is interesting to assess the reproducibility of the $M R$ protocol using the distributions obtained. As an example, in Figure 10A,B are plotted the thickness distribution of both left tibial compartments obtained at all time steps for one primate. As can be seen, the resulting distributions are well super-imposed. Figure 11A,B display the resulting means of lateral and medial distributions at all the time steps and their associated $95 \%$ CIs obtained by running one-way ANOVAs at all time steps and applying Tukey's post-hoc analysis on data from this single primate. The span of the vertical axis is set to $0.09 \mathrm{~mm}$ equal to bin sizes used in the quantification procedure. Twelve out of 20 of the comparisons yielded $\mathrm{P}$ value $<0.05$ indicating significantly different distribution means. This procedure was repeated for each primate and the results of the 104 tests (for two primates, acquisitions at D180 were not exploitable) are resumed in Table 2 (second line). Three categories were distinguished as follows: $30 \%$ of the comparisons yielded a $\mathrm{P}$ value above 0.05 (no statistically significant difference), $66 \%$ a $\mathrm{P}$ value under 0.05 but a $95 \%$ CI for the difference of the means under $0.09 \mathrm{~mm}$ and for only $4 \%$ were the $\mathrm{P}$ value under 0.05 and had a CI above $0.09 \mathrm{~mm}$ (columns 2-4 in Table 2). To put these results in perspective the same statistical tests were run at each time step but primate-wise. The results of the 132 inter-primate comparisons are also resumed in Table 2 $\left(3^{\text {rd }}\right.$ line $)$ whereby for the same classification we had 


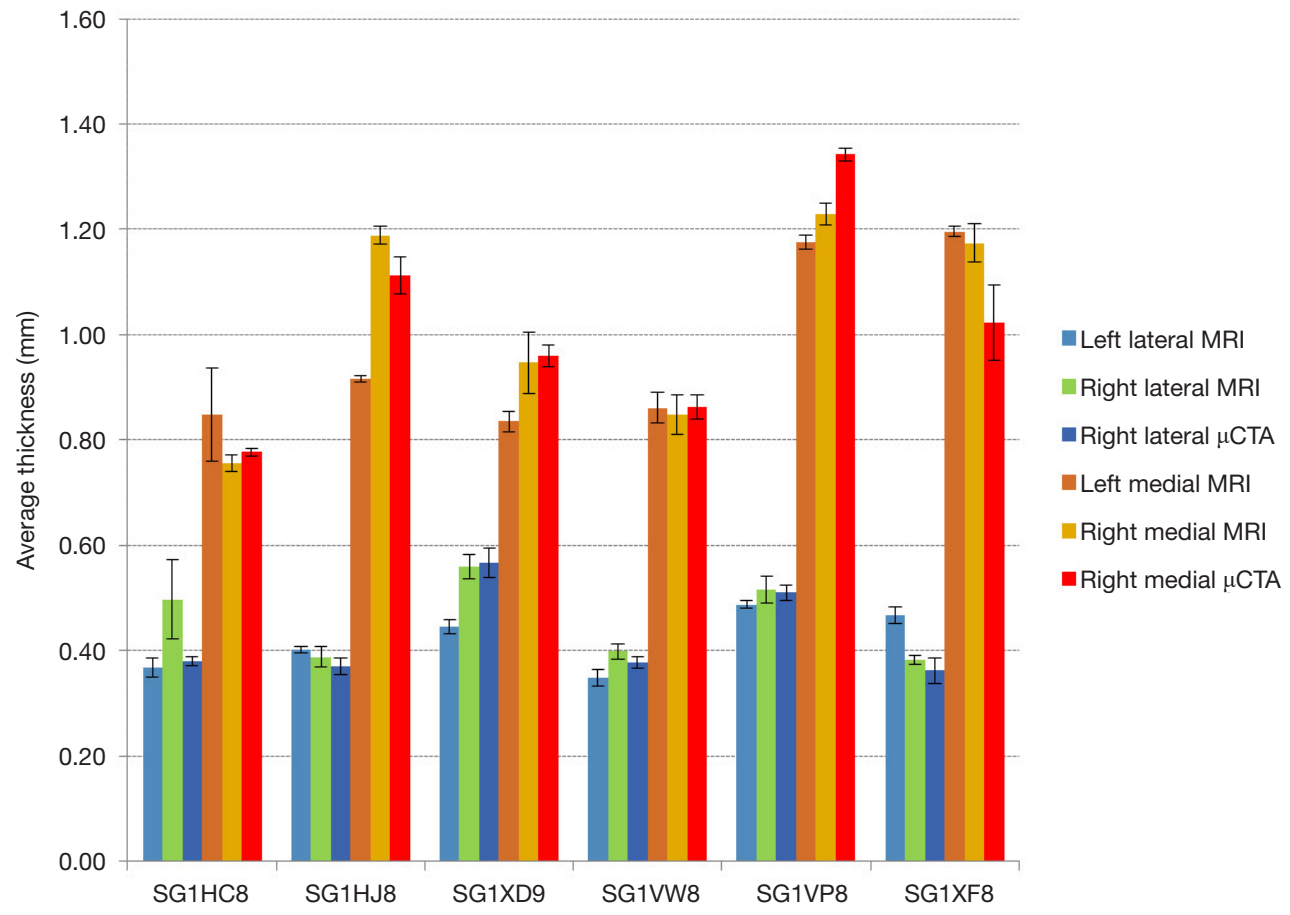

Figure 8 Time-based average of the mean thickness of left and right tibial compartments for all primates obtained by MRI and $\mu \mathrm{CTA}$. Error bars correspond to the SDs of the calculated mean thickness over the five-time steps. MRI, magnetic resonance imaging; $\mu$ CTA, microcomputed tomography arthrography; SD, standard deviation.
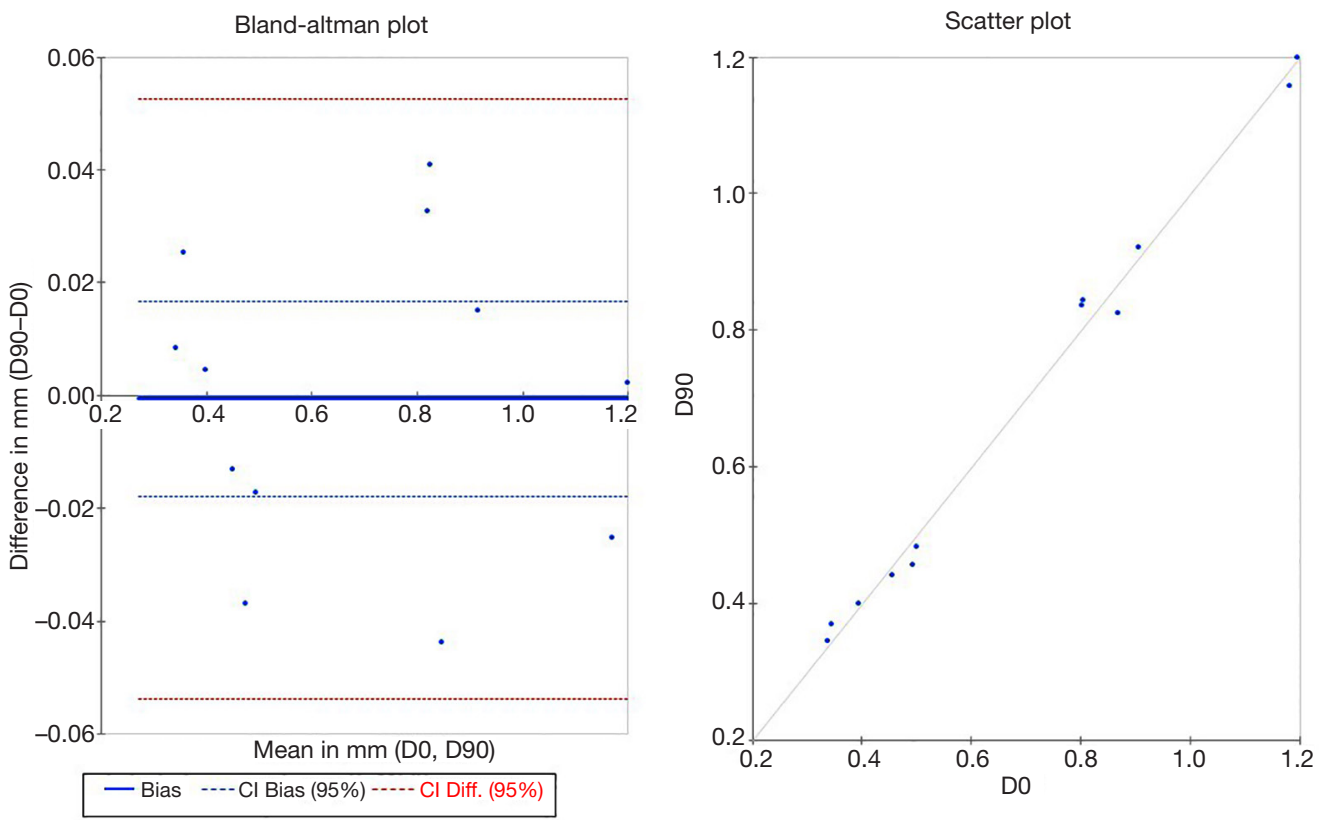

Figure 9 Bland-Altman and scatter plot of the mean thicknesses of lateral and medial cartilage obtained at day 0 (D0) and day 90 (D90). 
Table 1 Summary of Bland-Altman comparison of mean lateral and medial left tibial cartilage thicknesses obtained on all primates at different times steps through MRI

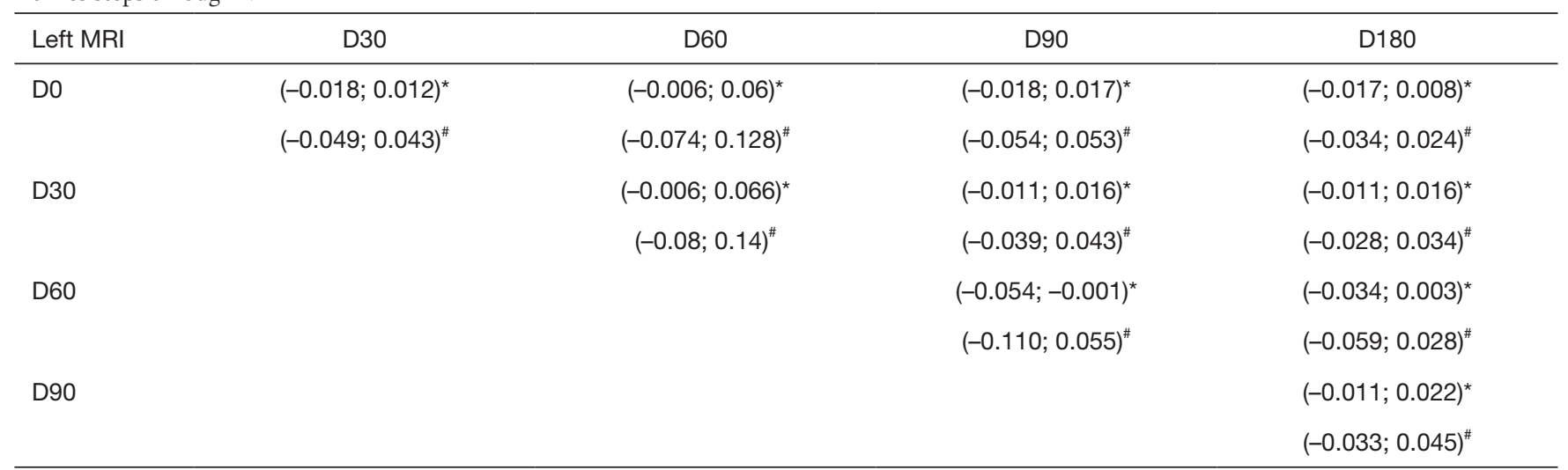

With * are given the $95 \% \mathrm{Cls}$ for the bias and with ${ }^{*}$ the $95 \% \mathrm{Cls}$ for the differences between paired values. MRI, magnetic resonance imaging; $\mathrm{Cl}$, confidence interval.
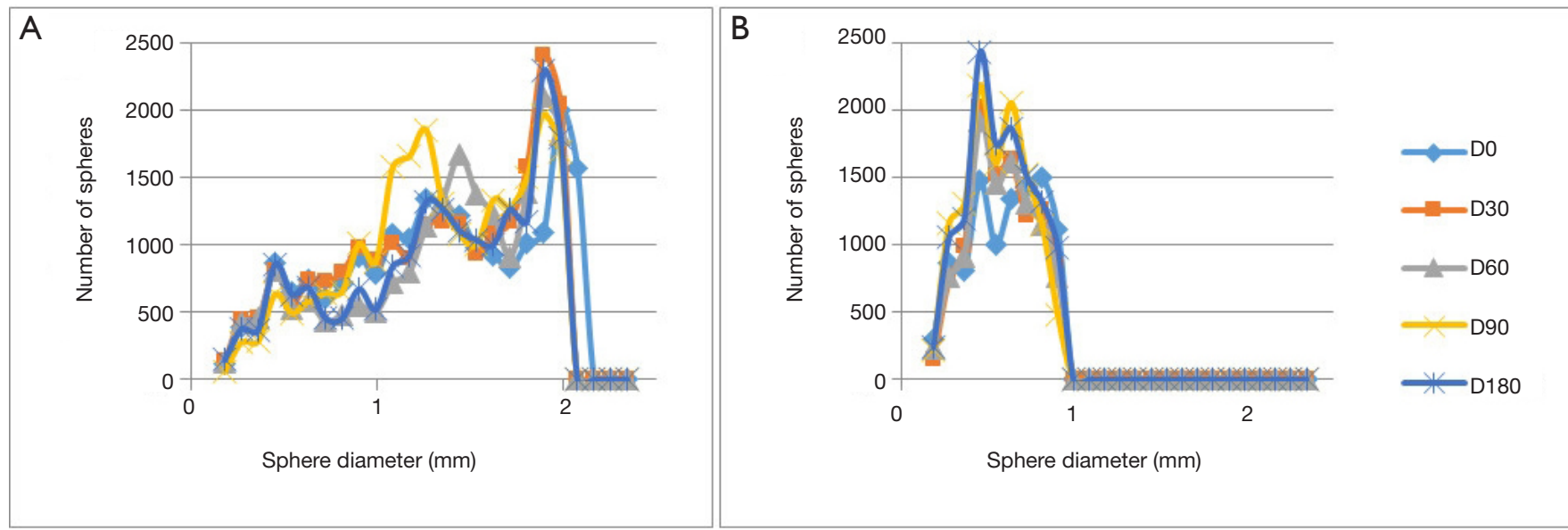

Figure $103 \mathrm{D}$ thickness parameter distributions obtained through the quantification procedure: (A) left medial and (B) left lateral tibial compartment obtained by MRI for a single primate (SG1XF8) at all time steps. MRI, magnetic resonance imaging.

respectively $11 \%, 39 \%$ and $51 \%$ (the sum exceeds $100 \%$ due to round-off).

\section{Evaluation of the impact of the $\mu C T A$ protocol}

Since the $\mu \mathrm{CTA}$ protocol requires contrast agent injection, it is important to assess whether this procedure induces a bias on the obtained results. The results obtained through MRI on the right knee (only this knee underwent the $\mu$ CTA protocol) at different time steps were first quantified. The resulting distributions were then compared using the same statistical test, thus yielding 120 two-by-two comparisons for the difference of the mean. These are summarized in
Table 2 ( $4^{\text {th }}$ line). Using the same classification, we obtained respectively $18 \%$ comparisons with a $\mathrm{P}$ value above 0.05 , $77 \%$ with a $\mathrm{P}$ value under 0.05 but a CI under $0.09 \mathrm{~mm}$ and only $5 \%$ a CI above $0.09 \mathrm{~mm}$.

The time-based averages of the mean thickness of right medial and lateral compartments through MRI were computed and displayed in Figure 8 . In this case, the maximum relative SD obtained was of $15 \%$.

Bland-Altman analyses were also carried out on the mean thicknesses of lateral and medial tibial cartilages of the right knee through MRI. A summary of the obtained CIs for the bias (with *) and the differences of paired values (with *) is given in Table 3. One of the comparisons yielded a CI for 

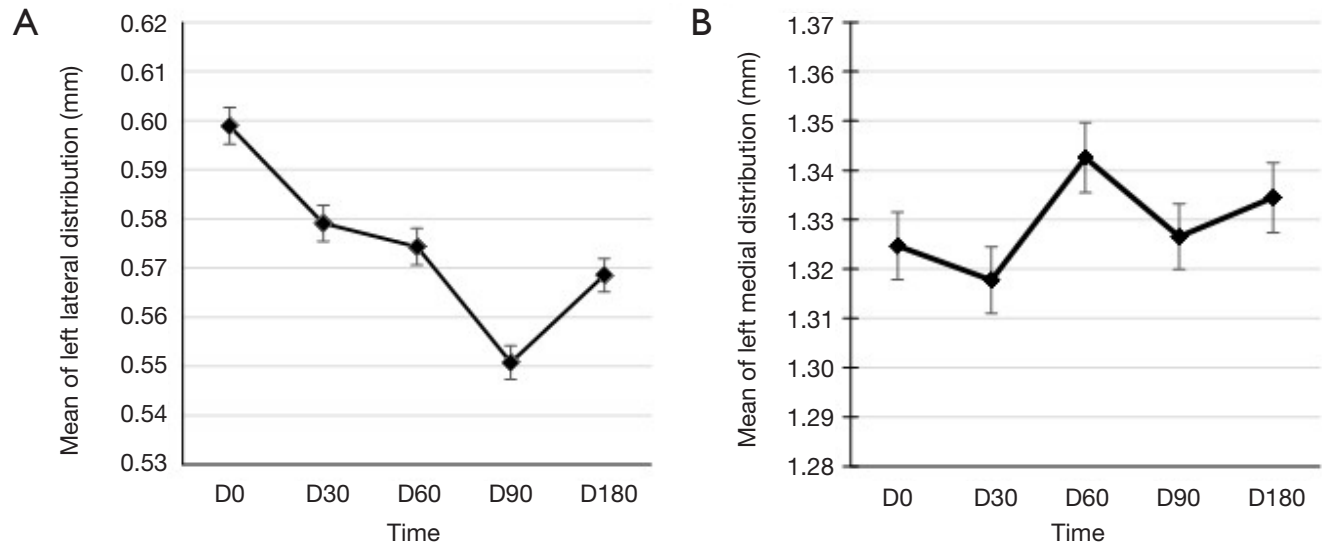

Figure 11 Plot of the means of the left (A) lateral and (B) medial tibial distributions obtained by MRI for one primate over the five-time steps. Vertical bars correspond to the CIs obtained by running Tukey's post-hoc analysis following a one-way ANOVA. MRI, magnetic resonance imaging; CI, confidence interval.

Table 2 Summary of the one-way ANOVAs with Tukey's post-hoc analysis carried out on different distributions obtained

\begin{tabular}{lllc}
\hline Compared data & $\mathrm{P}>\alpha$ & $\mathrm{P}<\alpha, \overline{C I}<0.09 \mathrm{~mm}$ & $\mathrm{P}<\alpha, \overline{C I}>0.09 \mathrm{~mm}$ \\
\hline MRI, left compartment, inter time-step for each primate [104] & $30 \%[31]$ & $66 \%[69]$ & $3 \%[4]$ \\
MRI, left compartment, inter-primate for each time-step [132] & $11 \%[14]$ & $39 \%[51]$ & $51 \%[67]$ \\
MRI, right compartment, inter time-step for each primate [120] & $18 \%[22]$ & $77 \%[92]$ & $5 \%[6]$ \\
MCTA, right compartment, inter time-step for each primate [120] & $11 \%[13]$ & $83 \%[100]$ & $6 \%[7]$ \\
\hline
\end{tabular}

Left (MRI only) and right (MRI and $\mathrm{CCTA}$ ) tibial compartment by comparing either for each primate the different measurements carried out at different time steps or for each time step the different measurements obtained on all primates. In each case is indicated the percentage as well as the corresponding number (in parentheses) of two-by-two comparisons yielding the given result. MRI, magnetic resonance imaging; $\mu \mathrm{CTA}$, micro-computed tomography arthrography.

Table 3 Summary of Bland-Altman comparisons of mean lateral and medial right tibial cartilage thicknesses obtained on all primates at different times steps through MRI

\begin{tabular}{|c|c|c|c|c|}
\hline Right MRI & D30 & D60 & D90 & D180 \\
\hline DO & $(-0.093 ; 0.14)^{\#}$ & $(-0.106 ; 0.157)^{\#}$ & $(-0.102 ; 0.142)^{\#}$ & $(-0.094 ; 0.113)^{\#}$ \\
\hline \multirow[t]{2}{*}{ D30 } & & $(-0.033 ; 0.031)^{\star}$ & $(-0.034 ; 0.018)^{\star}$ & $(-0.049 ; 0.018)^{\star}$ \\
\hline & & $(-0.099 ; 0.097)^{\#}$ & $(-0.089 ; 0.073)^{\#}$ & $(-0.119 ; 0.088)^{\#}$ \\
\hline D60 & & & $(-0.093 ; 0.079)^{\#}$ & $(-0.078 ; 0.049)^{\#}$ \\
\hline \multirow[t]{2}{*}{ D90 } & & & & $(-0.021 ; 0.019)^{\star}$ \\
\hline & & & & $(-0.043 ; 0.039)^{\#}$ \\
\hline
\end{tabular}

With ${ }^{*}$ are given the $95 \% \mathrm{Cls}$ for the bias and with ${ }^{\#}$ the $95 \%$ Cls for the differences between paired values. MRI, magnetic resonance imaging; $\mathrm{Cl}$, confidence interval. 

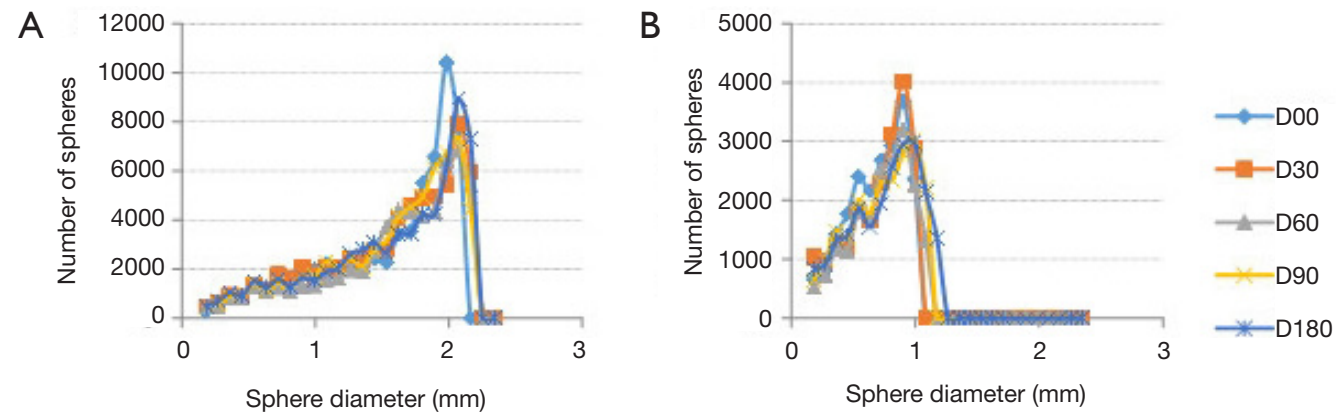

Figure 12 Thickness distribution of (A) right medial and (B) right lateral tibial compartment obtained by $\mu$ CTA for a single primate (SG1VP8) at all time steps. $\mu$ CTA, micro-computed tomography arthrography.

Table 4 Summary of Bland-Altman comparison of mean lateral and medial right tibial cartilage thicknesses obtained on all primates at different times steps through $\mu \mathrm{CTA}$

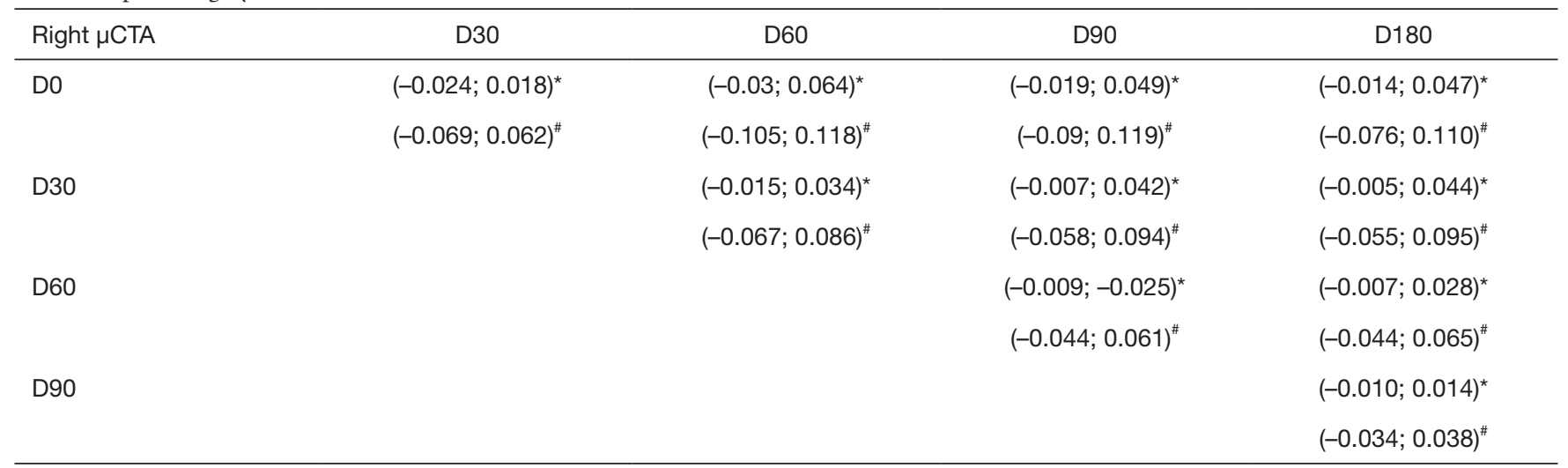

With ${ }^{*}$ are given the $95 \% \mathrm{Cls}$ for the bias and with ${ }^{*}$ the $95 \% \mathrm{Cls}$ for the differences between paired values. $\mu \mathrm{CTA}$, micro-computed tomography arthrography; $\mathrm{Cl}$, confidence interval.

the bias excluding zero (D60 vs. D90) while seven had a CI for the differences between paired values above $0.09 \mathrm{~mm}$.

\section{Evaluation of results obtained by $\mu C T A$}

The final objective of this study was to assess $\mu$ CTA for cartilage morphology imaging. The first task is to evaluate the reproducibility of data obtained through $\mu$ CTA. The obtained distributions for one primate is shown in Figure 12. Similar statistical tests carried out on the resulting distributions for all primates across the different time-steps yielded the results summarized line 5 in Table 2 . Eleven percent comparisons with a $\mathrm{P}$ value above $0.05,83 \%$ had a P value under 0.05 but a CI under $0.09 \mathrm{~mm}$ and only $6 \%$ a CI above $0.09 \mathrm{~mm}$. The time-based averages of the mean thickness of right medial and lateral compartments through $\mu$ CTA were computed and displayed in Figure 8. In this case, the maximum relative SD obtained was of $7 \%$.

Bland-Altman analyses carried out on the mean thicknesses of lateral and medial tibial cartilages of the right knee through $\mu$ CTA are summarized in Table 4. One of the comparisons yielded a CI for the bias excluding zero while five had a CI for the differences between paired values above $0.09 \mathrm{~mm}$.

MRI and $\mu$ CTA distributions of the right compartments were then compared. For each primate, MR distributions obtained at a given time step were statistically tested ( $t$-test with Welch's correction to account for different variances of distributions) with the equivalent $\mu$ CTA distribution. Sixty two-by-two comparisons were carried out and the subsequent results which are summarized in Table 5 show that only $2 \%$ of the comparisons yielded a $\mathrm{P}$ value above the significance level to be removed from the manuscript with an additional $50 \%$ having a $\mathrm{P}$ value under the significance level and a $\mathrm{CI}$ of the 
Table 5 Summary of the one-way ANOVAs with Tukey's post-hoc analysis carried out on the right tibial compartments distributions obtained by comparing for a given primate and a given time-step MRI and $\mu$ CTA distributions

\begin{tabular}{lcccc}
\hline Compared data & $\mathrm{P}>\alpha$ & $\mathrm{P}<\alpha, \mathrm{Cl}<0.09 \mathrm{~mm}$ & $\mathrm{P}<\alpha, \mathrm{Cl} \in[0.09 ; 0.18] \mathrm{mm}$ & $\mathrm{P}<\alpha, \mathrm{Cl}>0.18 \mathrm{~mm}$ \\
\hline $\begin{array}{l}\text { Inter-modality for each primate } \\
\text { at each time-step [60] }\end{array}$ & $2 \%[1]$ & $50 \%[30]$ & $45 \%[27]$ & $3 \%[2]$
\end{tabular}

In each case is indicated the percentage as well as the corresponding number (in parentheses) of two-by-two comparisons yielding the given result. MRI, magnetic resonance imaging; $\mu \mathrm{CTA}$, micro-computed tomography arthrography.

Table 6 Comparison of mean lateral and medial right tibial cartilage thicknesses obtained on all primates at similar times steps obtained through $\mu \mathrm{CTA}$ and MRI

\begin{tabular}{lccccc}
\hline Compared data & D0 & D30 & D60 & D90 & D180 \\
\hline Right $\mu$ CTA vs. MRI & $(-0.084 ; 0.028)^{*}$ & $(-0.098 ; 0.019)^{\star}$ & $(-0.078 ; 0.02)^{\star}$ & $(-0.056 ; 0.029)^{\star}$ & $(-0.048 ; 0.04)^{\star}$ \\
& $(-0.181 ; 0.125)^{\#}$ & $(-0.219 ; 0.140)^{\#}$ & $(-0.179 ; 0.121)^{\#}$ & $(-0.145 ; 0.117)^{\#}$ & $(-0.141 ; 0.132)^{\#}$ \\
\hline
\end{tabular}

With * are given the $95 \% \mathrm{Cls}$ for the bias and with " the $95 \%$ Cls for the differences between paired values. MRI, magnetic resonance imaging; $\mu \mathrm{CTA}$, micro-computed tomography arthrography; $\mathrm{Cl}$, confidence interval.

difference of the means under $0.09 \mathrm{~mm}$. Of the remaining $48 \%$ having a CI above $0.09 \mathrm{~mm}, 45 \%$ had a CI ranging between 0.09 and $0.18 \mathrm{~mm}$ and $3 \%$ above $0.18 \mathrm{~mm}$.

Finally, the mean thicknesses of lateral and medial tibial cartilages of the right knee obtained through $\mu$ CTA and MRI at similar time-steps are compared with Bland-Altman analyses and the resulting CIs for the bias (with *) and the difference of the paired values (with ${ }^{\#}$ ) are summarized in Table 6. None of the five comparisons yielded a CI for the bias which didn't include zero. However, all displayed a CI for the difference of paired values which exceeded $0.09 \mathrm{~mm}$.

\section{Discussion}

\section{MRI protocol}

The development of the home-made coil was a starting point towards enabling the application of the MRI protocol on the NHP knees. Indeed, in addition to providing highresolution images, it also allowed for a reasonable scan time which is an important factor to take into consideration if one wants to follow ethical guidelines. The use of the susceptibility matching foam was also of equal importance since without it shimming on both knees was nearly impossible and thus simultaneous acquisitions of both NHP knees would have been impossible. Both factors played an important role in rendering the acquisitions possible within the set time frame.

Considering results obtained on the $3 \mathrm{D}$ thickness parameter distributions, Figure $7 A, B$ report the thickness distributions of both left compartments for all primates at baseline. Two sub-groups appear considering maximum cartilage thickness in either compartment. The same can be deduced from Figure 8 and is weight-related; SGIVP8 and SG1XF8 had the highest weights. This is also pointed out when considering the inter-primate statistical tests (line 3, Table 2). Among the 67 comparisons yielding $\mathrm{P}>0.05$ and $\overline{C I}>0.09 \mathrm{~mm}, 58$ are due to differences between SG1VP8 and SG1XF8 and the rest of the group.

On an individual basis, the resulting distributions at different time-steps for the left compartments have comparable shapes as can be seen in Figure 10A,B. Statistical analysis carried out confirms this visual impression: Figure 11 shows the difference of the means of the distributions are all well below the resolution of the quantification procedure $(0.09 \mathrm{~mm})$ and in-plane pixel size of MR images although statistical tests point out a significant difference. Extending to all primates resulted in the same trend (Table 2, line 2) since a vast majority (96\%) of the tests yielded either $\mathrm{P}>\alpha$ or $\overline{C I}<0.09 \mathrm{~mm}$.

Bland-Altman analyses (Table 1) carried out on the mean of left lateral and medial tibial thicknesses also follow the same trend. With only mean values at D60 and D90 having a CI for the bias excluding zero. Considering the CI of paired values, three comparisons had a limit above $0.09 \mathrm{~mm}$ which was the bin size of our segmentation procedure. However, considering that the actual voxel size of the MR images was $112 \times 131 \times 220 \mathrm{\mu m}^{3}$, then it is reasonable to assume that $0.09 \mathrm{~mm}$ is too restrictive and that $0.18 \mathrm{~mm}$ would be a more adapted threshold. In this case none of the 
CIs had a limit exceeding this value.

We can thus conclude that our results present very little intra-individual variability also meaning that primates as from 4 years old have stable cartilages within the following 6 months (and probably after). The reproducibility of our procedure was also highlighted by the relative SD obtained which is less or equal to $10 \%$. The protocol can hence be said to deliver sufficiently reliable results to be used for further investigation.

\section{Evaluation of the impact of the $\mu$ CTA protocol}

An examination of results obtained on the right knee through MRI can allow us to have a very good insight on the impact of the $\mu \mathrm{CTA}$ protocol. The later requiring injection of a contrast agent, it was necessary to check that neither the injection procedure nor the contrast agent produce side-effects to the visible cartilage structure. MRI on the right knee was carried out after $\mu \mathrm{CTA}$ at all the time steps. ANOVAs on the obtained distributions (Table 2, line $4)$ indicate that there was no difference in results compared to the left knee; only $5 \%$ (6 out of the 120 comparisons) exhibited significant differences of the means of the distributions (for the left knee it was 4\%).

This is reinforced when comparing the time-based average of the mean thicknesses of left and right tibial compartments measured by MRI and shown in Figure 6. Left and right values although unequal are all independently consistent as can be seen with the small SDs which are generally equivalent in both compartments. Bland-Altman analyses display only one CI for the bias excluding zero. Coincidentally it concerned the same two time points (D60 and D90) as for the left compartment but this cannot be accounted for at present. None of the CIs for the difference of paired values had a limit exceeding $0.18 \mathrm{~mm}$ similarly to the left knee. This indicates that there is no increased variability on the right knee due to the $\mu \mathrm{CTA}$ protocol. SG1HJ8 is an outlier with average values of left and right medial tibial cartilage well apart (respectively 0.92 and $1.19 \mathrm{~mm}$ ). This difference has not yet been accounted for.

\section{Evaluation of $\mu C T A$}

In the previous section, it was shown that the $\mu \mathrm{CTA}$ injection protocol was bias-free. Comparing the distributions and mean cartilage thicknesses obtained at different time-steps through $\mu \mathrm{CTA}$ will test reproducibility of $\mu$ CTA on NHP for tibial cartilage imaging. This was verified when considering for example Figure 12 which shows $\mu$ CTA thickness distributions of the right compartments at different time-steps for one primate. Overall qualitative impression is that of well super-imposed distributions leading us to believe that the $\mu \mathrm{CTA}$ imaging procedure produced consistent results. This is quantitatively supported by the one-way ANOVAs carried out and whose results are shown in Table 2 (line 5). Although the means of the distributions are statistically significantly different, the differences are well under the set $90 \mu \mathrm{m}$ level. An overwhelming majority (94\%) do not display significant differences of the means above $90 \mu \mathrm{m}$. This is comparable to MRI results with respectively $96 \%$ and $95 \%$ for the left and right compartments.

Considering the mean thickness of each compartment calculated from $\mu \mathrm{CTA}$ images, time-based average for each primate (Figure 8) displayed SDs comparable to the MRIvalues; in the medial compartment, maximum SD was of $0.059 \mathrm{~mm}$ for MRI and slightly higher to $0.072 \mathrm{~mm}$ for $\mu \mathrm{CTA}$. On the lateral compartment, it was the opposite with 0.076 and $0.028 \mathrm{~mm}$ respectively for MRI and $\mu \mathrm{CTA}$. In any case, the SDs can be accounted for by the quantification procedure. Bland-Altman analyses (Table 4) also support this statement since 9 out of 10 comparisons had a CI for the bias including zero. It should be noted that the one which didn't fall into this category was again D60 vs. D90. As for the CI for differences between paired values, none had limits above $0.18 \mathrm{~mm}$.

Comparing results obtained through the two modalities, the time-based averages obtained for individual primates showed some discrepancies. For instance, in the medial compartment, the maximum difference was of $0.15 \mathrm{~mm}$ (SG1XF8) whereas in the lateral compartment, it was of $0.12 \mathrm{~mm}$ (SG1HC8). Bland-Altman comparisons (Table 6) display CIs for the bias which all include zero however the limits are higher than in all the other three situations (timewise left MRI, right MRI and right $\mu \mathrm{CTA}$ ). Moreover, there is a slight tendency for theses CI to be centered on negative values particularly for the earlier time steps but of course the small number of samples doesn't enable further conclusions. Time-based averages of $\mu$ CTA values for the mean cartilage thickness in the tibial compartment tend to be smaller than MR values (Figure 8) as has been also observed in another study on cadaveric knees (32). As for the CIs for the difference between paired values obtained through Bland-Altman analyses (Table 6), one can notice that among the five comparisons, two have limits which exceed $0.18 \mathrm{~mm}$. 
A further analysis of MRI and $\mu$ CTA distributions of the right compartments (Table 5) show that nearly half (48\%) had CIs above the $90 \mu \mathrm{m}$. One of the potential reasons might be the timing of $\mu \mathrm{CTA}$ with respect to the injection of the contrast agent. Since the contrast agent can diffuse inside the cartilage structure, this can lead to a reduced measured cartilage thickness. Another possible explanation comes from the unequal resolutions of MRI and $\mu \mathrm{CTA}$ acquisitions. Voxel sizes in MRI are bigger than that of $\mu \mathrm{CTA}$ and the quantification procedure was carried out with a common bin size equal to that of $\mu \mathrm{CTA}$ acquisitions. It is plausible that in this context, the quantification procedure for MRI images will lead to distributions with higher means. It can also be argued that if the threshold was set to $0.18 \mathrm{~mm}$ which is reasonable with respect to the bin sizes and voxels sizes of MRI images, the proportion of statistical comparisons pointing to a difference between $\mu \mathrm{CTA}$ and MRI distributions decreases drastically to a mere $3 \%$ as can be seen from Table 5 .

Finally, it is also interesting to take note of some characteristic values of the tibial cartilage of primates. The maximum thickness measured in the medial compartment is equal to $2.1 \mathrm{~mm}(\mathrm{MRI})$ and $2.2 \mathrm{~mm}(\mu \mathrm{CTA})$ whereas in the lateral compartment it is equal to $1.0 \mathrm{~mm}$ (MRI) and $1.2 \mathrm{~mm}(\mu \mathrm{CTA})$. Mean thicknesses measured are equal to $1.0 \mathrm{~mm}$ for the medial section and $0.4 \mathrm{~mm}$ for the lateral section (MRI and $\mu \mathrm{CTA}$ ). This places the mean tibial cartilage thickness of NHP in between those of small animals like mice, rats or guinea pigs (mean thicknesses are respectively of the order of 30, 200 and $300 \mu \mathrm{m}$ ) and that of humans where the mean thickness is around $2 \mathrm{~mm}$. These values represent tangible targets for all imaging protocols carried out on NHP.

\section{Conclusions}

In this study, a dedicated MR protocol using standard imaging to acquire high spatial resolution $\left(112 \times 131 \mu \mathrm{m}^{2} / 220 \mu \mathrm{m}\right.$ thickness) images of knee cartilage of NHPs in a reasonable scan time (20 minutes) was developed and validated. Key points in enabling this was the use of a dedicated homemade coil and susceptibility matching foam for knee positioning. A second imaging protocol using $\mu \mathrm{CTA}$ enabled fast (15 minutes) and relevant (90 $\mu \mathrm{m}$ isotropic resolution) acquisitions of the NHP knees also allowing to study its $3 \mathrm{D}$ cartilage layer.

Tibial cartilage images from those two modalities were then compared on six NHP models during a 180 days protocol with five examinations (D0, D30, D60, D90 and D180) for both knees (MRI for both knees and $\mu$ CTA for the right). From the acquired images, it was possible to segment tibial cartilage and deduce bin distributions characterizing them and then deduce mean cartilage thicknesses of lateral and medial tibial compartments. These were used to statistically test the reproducibility of the MR and $\mu$ CTA protocols, whether the $\mu$ CTA protocol was bias-free and lastly the consistency of MR and $\mu \mathrm{CTA}$ based values.

The first three points were clearly positive meaning that both modalities together with their developed experimental setup were valuable to measure cartilage morphology in NHP models. For the last question, results from MR and $\mu$ CTA were coherent but a slight tendency for smaller $\mu C T A$ based values compared to MRI was observed but can only be confirmed using additional subjects.

In any case, this work shows the possibility of using NHP in designing follow-up studies focusing on OA by for example inducing it. On the MRI side, using these models, it would be interesting to develop and test sequences such as T2 mapping, T1rho or dipolar contrast to assess their sensitivity to earlier signs of cartilage degradations.

\section{Acknowledgments}

Funding: This work was supported by OSEO by grant E! 5671 from the Eurostars European program and performed within the framework of the LABEX PRIMES (ANR-11LABX-0063) of Université de Lyon, within the program "Investissements d'Avenir" (ANR-11-IDEX-0007) operated by the French National Research Agency (ANR).

\section{Footnote}

Conflicts of Interest: All authors have completed the ICMJE uniform disclosure form (available at http://dx.doi. org/10.21037/qims-20-116). Dr. EC reports grants from European Commission - Eurostars co-funding, during the conduct of the study; other from Voxcan, from European Commission or French Research Ministry, personal fees from National Veterinary School of Lyon - VetAgro-Sup, outside the submitted work; and Voxcan is a CRO who sells preclinical studies in the domain of osteoarthritis to its clients. Drs. KTVK and OB report grants from Universite Lyon 1, during the conduct of the study. Drs. FT and HC 
report grants from European Commission - Eurostars cofunding, during the conduct of the study. Dr. LM reports grants from European Commission - Eurostars co-funding, during the conduct of the study; other from Voxcan, from European Commission or French Research Ministry, outside the submitted work. The other authors have no conflicts of interest to declare.

Ethical Statement: The authors are accountable for all aspects of the work in ensuring that questions related to the accuracy or integrity of any part of the work are appropriately investigated and resolved. All animal studies and experiments were performed under project license $\mathrm{N}^{\circ} 1077$ granted by the Institutional Animal Care and Use Committee (IACUC) of VetAgro-Sup in compliance with the European regulation on animal experimentation (Directive 2010/63/EU) and its national transposition.

Open Access Statement: This is an Open Access article distributed in accordance with the Creative Commons Attribution-NonCommercial-NoDerivs 4.0 International License (CC BY-NC-ND 4.0), which permits the noncommercial replication and distribution of the article with the strict proviso that no changes or edits are made and the original work is properly cited (including links to both the formal publication through the relevant DOI and the license). See: https://creativecommons.org/licenses/by-nc-nd/4.0/.

\section{References}

1. Centers for Disease Control and Prevention (CDC). Prevalence of disabilities and associated health conditions among adults--United States, 1999. MMWR Morb Mortal Wkly Rep 2001;50:120-5. Erratum in: MMWR Morb Mortal Wkly Rep 2001;50:149.

2. Felson DT, Lawrence RC, Dieppe PA, Hirsch R, Helmick CG, Jordan JM, Kington RS, Lane NE, Nevitt MC, Zhang Y, Sowers M, McAlindon T, Spector TD, Poole AR, Yanovski SZ, Ateshian G, Sharma L, Buckwalter JA, Brandt KD, Fries JF. Osteoarthritis: new insights. Part 1: the disease and its risk factors. Ann Intern Med 2000;133:635-46.

3. Felson DT. The sources of pain in knee osteoarthritis. Curr Opin Rheumatol 2005;17:624-8.

4. Bennell K, Hinman R. Exercise as a treatment for osteoarthritis. Curr Opin Rheumatol 2005;17:634-40.

5. Hunter DJ, Roemer FW, Mosher TJ. A special osteoarthritis and cartilage issue on imaging in osteoarthritis. Osteoarthritis Cartilage 2014;22:1347-8.

6. Dunlop DD, Manheim LM, Song J, Chang RW. Arthritis prevalence and activity limitations in older adults. Arthritis Rheum 2001;44:212-21.

7. Murphy L, Schwartz TA, Helmick CG, Renner JB, Tudor G, Koch G, Dragomir A, Kalsbeek WD, Luta G, Jordan JM. Lifetime risk of symptomatic knee osteoarthritis. Arthritis Rheum 2008;59:1207-13.

8. Teichtahl AJ, Wluka AE, Davies-Tuck ML, Cicuttini FM. Imaging of knee osteoarthritis. Best Pract Res Clin Rheumatol 2008;22:1061-74.

9. Hunter DJ, Le Graverand MP, Eckstein F. Radiologic markers of osteoarthritis progression. Curr Opin Rheumatol 2009;21:110-7.

10. Braun HJ, Gold GE. Diagnosis of osteoarthritis: imaging. Bone 2012;51:278-88.

11. Eckstein F, Adam C, Sittek H, Becker C, Milz S, Schulte E, Reiser M, Putz R. Non-invasive determination of cartilage thickness throughout joint surfaces using magnetic resonance imaging. J Biomech 1997;30:285-9.

12. Cohen ZA, McCarthy DM, Kwak SD, Legrand P, Fogarasi F, Ciaccio EJ, Ateshian GA. Knee cartilage topography, thickness, and contact areas from MRI: in-vitro calibration and in-vivo measurements. Osteoarthritis Cartilage 1999;7:95-109.

13. Kraus VB. Biomarkers in osteoarthritis. Curr Opin Rheumatol 2005;17:641-6.

14. Bruyere O, Collette J, Kothari M, Zaim S, White D, Genant H, Peterfy C, Burlet N, Ethgen D, Montague T, Dabrowski C, Reginster JY. Osteoarthritis, magnetic resonance imaging, and biochemical markers: a one year prospective study. Ann Rheum Dis 2006;65:1050-4.

15. Eckstein F, Cicuttini F, Raynauld JP, Waterton JC, Peterfy C. Magnetic resonance imaging (MRI) of articular cartilage in knee osteoarthritis (OA): morphological assessment. Osteoarthritis Cartilage 2006;14 Suppl A:A46-75.

16. Ding C, Cicuttini F, Jones G. How important is MRI for detecting early osteoarthritis? Nat Clin Pract Rheumatol 2008;4:4-5.

17. Kotwal N, Li J, Sandy J, Plaas A, Sumner DR. Initial application of EPIC- $\mu \mathrm{CT}$ to assess mouse articular cartilage morphology and composition: effects of aging and treadmill running. Osteoarthritis Cartilage 2012;20:887-95.

18. Piscaer TM, Waarsing JH, Kops N, Pavljasevic P, Verhaar JA, van Osch GJ, Weinans $H$. In vivo imaging of cartilage degeneration using microCT-arthrography. Osteoarthritis 
Cartilage 2008;16:1011-7.

19. Xie L, Lin AS, Levenston ME, Guldberg RE. Quantitative assessment of articular cartilage morphology via EPICmicroCT. Osteoarthritis Cartilage 2009;17:313-20.

20. Goebel JC, Bolbos R, Pham M, Galois L, Rengle A, Loeuille D, Netter P, Gillet P, Beuf O, Watrin-Pinzano

A. In vivo high-resolution MRI (7T) of femoro-tibial cartilage changes in the rat anterior cruciate ligament transection model of osteoarthritis: a cross-sectional study. Rheumatology (Oxford) 2010;49:1654-64.

21. Bolbos R, Benoit-Cattin H, Langlois JB, Chomel A, Chereul E, Odet C, Pastoureau P, Janier M, Beuf O. Knee cartilage thickness measurements using MRI: a 4(1/2)-month longitudinal study in the meniscectomized guinea pig model of OA. Osteoarthritis Cartilage 2007;15:656-65.

22. Batiste DL, Kirkley A, Laverty S, Thain LM, Spouge AR, Gati JS, Foster PJ, Holdsworth DW. High-resolution MRI and micro-CT in an ex vivo rabbit anterior cruciate ligament transection model of osteoarthritis. Osteoarthritis Cartilage 2004;12:614-26.

23. Batiste DL, Kirkley A, Laverty S, Thain LM, Spouge AR, Holdsworth DW. Ex vivo characterization of articular cartilage and bone lesions in a rabbit ACL transection model of osteoarthritis using MRI and micro-CT. Osteoarthritis Cartilage 2004;12:986-96.

24. Carlson CS, Loeser RF, Jayo MJ, Weaver DS, Adams MR, Jerome CP. Osteoarthritis in cynomolgus macaques: a primate model of naturally occurring disease. J Orthop Res 1994;12:331-9.

25. Carlson CS, Loeser RF, Purser CB, Gardin JF, Jerome CP. Osteoarthritis in cynomolgus macaques. III: Effects of age, gender, and subchondral bone thickness on the severity of disease. J Bone Miner Res 1996;11:1209-17.

26. Jerome CP, Peterson PE. Nonhuman primate models in skeletal research. Bone 2001;29:1-6.

27. Buckwalter JA, Martin JA, Olmstead M, Athanasiou $\mathrm{KA}$, Rosenwasser MP, Mow VC. Osteochondral repair

Cite this article as: Tse Ve Koon K, Grenier D, Taborik F, Perrier AL, Mahieu-Williame L, Magnier L, Chuzel T, Contamin H, Chereul E, Beuf O. Comparison of highresolution magnetic resonance imaging and micro-computed tomography arthrography for in-vivo assessment of cartilage in non-human primate models. Quant Imaging Med Surg 2021;11(8):3431-3447. doi: 10.21037/qims-20-116 of primate knee femoral and patellar articular surfaces: implications for preventing post-traumatic osteoarthritis. Iowa Orthop J 2003;23:66-74.

28. Ogawa S, Awaga Y, Takashima M, Hama A, Matsuda A, Takamatsu H. Knee osteoarthritis pain following medial meniscectomy in the nonhuman primate. Osteoarthritis Cartilage 2016;24:1190-9.

29. Stone AV, Vanderman KS, Willey JS, Long DL, Register TC, Shively CA, Stehle JR Jr, Loeser RF, Ferguson $\mathrm{CM}$. Osteoarthritic changes in vervet monkey knees correlate with meniscus degradation and increased matrix metalloproteinase and cytokine secretion. Osteoarthritis Cartilage 2015;23:1780-9.

30. Michalak GJ, Walker R, Boyd SK. Concurrent assessment of cartilage morphology and bone microarchitecture in the human knee using contrast-enhanced HR-pQCT imaging. J Clin Densitom 2019;22:74-85.

31. Hildebrand T, Rüegsegger P. A new method for the model-independent assessment of thickness in threedimensional images. J Microsc 1997;185:67-75.

32. Perrier AL, Chereul E, Grenier D, Taborik F, MahieuWilliame L, Abdallah M, Chuzel T, Martin S, Magnier L, Goebel JC, Pesesse X, Pietri S, Contamin H, Beuf O. Morphological assessment of non-human primate model of osteoarthritis: comparison of HR-MRI with CT arthrography (CTA). Melbourne: ISMRM 20th Annual Meeting \& Exhibition, 2012.

33. Lee GC, Goodwill PW, Phuong K, Inglis BA, Scott GC, Hargreaves BA, Li L, Chen AC, Shah RN, Conolly SM. Pyrolytic graphite foam: a passive magnetic susceptibility matching material. J Magn Reson Imaging 2010;32:684-91.

34. Lee G, Jordan C, Tiet P, Ruiz C, McCormick J, Phuong K, Hargreaves B, Conolly S. Improved frequency selective fat suppression in the posterior neck with tissue susceptibility matched pyrolytic graphite foam. J Magn Reson Imaging 2015;41:684-93. 